\title{
41 Year Old Woman with FeVErs and Shortness of Breath
}

\author{
Jonathan Jun, $M D$
}

A 41 year-old African American female with a history of asthma, hypertension, and schizophrenia presented to the ER in February 2005 with shortness of breath, wheezing, and a "wet-sounding" but nonproductive cough. She reported chills, subjective fever, rhinorrhea, and malaise for five days prior. Her only medication was albuterol, which she normally used twice weekly as needed. She was never intubated for asthma. On review of systems, she had progressive dyspnea on exertion of less than two blocks for the last year. She denied night sweats, weight loss, or sick contacts, HIV exposure, alcohol or drug abuse. She admitted to smoking 1 pack per day for more than 15 years.

On physical exam, her temperature was $97.0 \mathrm{~F}$, pulse 96 beats per minute, respirations 22 breaths per minute, blood pressure 130/100 $\mathrm{mmHg}$. She had an oxygen saturation of $77 \%$ on room air which improved to $93 \%$ on 4 liters oxygen. She was an obese female in moderate respiratory distress. Her orophaynx was clear. Her neck was supple without thyromegaly or lymphadenopathy. Jugular venous distension measured at $10 \mathrm{~cm}$ above the Angle of Louis. Heart exam revealed tachycardia without murmur, rub or gallop. Lungs had scattered rhonchi and wheezes bilaterally. Faint crackles were audible at the bases. She had trace symmetrical ankle edema.

Laboratory data on admission showed a WBC of 5900/L, (mostly neutrophils and 30\% lymphocytes) hemoglobin $14.7 \mathrm{~g} / \mathrm{dL}$, platelets $142 \mathrm{k} / \mathrm{L}$. Chemistries were grossly normal except for a bicarbonate of $31 \mathrm{mmol} / \mathrm{L}$. ABG on $4 \mathrm{~L} \mathrm{O} 2$ showed $\mathrm{pH}$ 7.32, PCO2 of 31, $\mathrm{PaO} 2$ 71. LDH level was elevated at 687. Her coagulation indices, liver function, cardiac enzymes, and urinalysis were within normal limits. Chest X-ray showed a right lower-lobe consolidation and diffuse infiltrates bilaterally. A chest CT further revealed hilar adenopathy and a small right pleural effusion while excluding pulmonary embolism (Figure 1). Blood cultures were sent, ceftriaxone and azithromycin were administered, and serial nebulizer treatments were given for her asthma.

The patient eventually became more hypoxic and was moved to the ICU where she was intubated. Pressor agents were required for hypotension, and her degree of hypoxia required an $\mathrm{FiO} 2$ of $100 \%$ with +15 of PEEP. On her subsequent chest radiographs, she developed diffuse alveolar infiltrates in multiple areas consistent with ARDS. Bronchoscopy and thoracentesis did not elicit any bacterial, fungal, or neoplastic etiology for her respiratory failure. Silver stain for PCP was negative. HIV serology was negative.

She remained intubated and on pressors for over 1 week. During this time, admission influenza titers came back elevated for both influenza A and B (Each was 1.6 with a reference range $<0.20$ ) Viral culture from BAL later grew influenza A.

\section{Discussion}

At first glance this case appeared to be one of bacterial pneumonia, sepsis and ARDS. The lobar consolidation on her X-ray was more consistent with bacterial pneumonia. Her septic hemodynamics also suggested a bacterial infection (although influenza mimicking septic shock has been reported in very young patients) Therefore, it is not clear if her critical illness was due solely to influenza. More likely, she suffered from influenza complicated by secondary bacterial pneumonia. Regardless, her case illustrates how quickly and severely a patient with underlying lung disease (which probably was more severe than she reported, given findings of cor pulmonale and compensated respiratory acidosis) can deteriorate when infected with influenza. Clinical awareness is paramount to timely diagnosis, prophylaxis, and effective treatment.

In the northern hemisphere, influenza epidemics occur yearly from December to April. Normally the illness is most severe in the elderly, very young, or those with chronic medical problems. Uncomplicated illness typically resolves within 1 week, but can progress to primary influenza viral pneumonia or secondary bacterial pneumonia in susceptible persons. The latter occurs as the virus denudes ciliated epithelium, allowing bacterial entry into the lower respiratory tract. Streptococcus pneumoniae, staphylococcus aureus, and Haemophilus influenza are the most common pathogens isolated in this setting.

As was demonstrated in this case, serologic influenza tests, while relatively sensitive and specific, do not generally yield data within a clinically relevant time period. A diagnostic test should produce a fourfold or greater rise in antibody titers between acute illness and the convalescent phase approximately 10 days later. Our patient did not have such a follow-up titer drawn, and diagnosis was based upon viral culture. Cultures could also be obtained by throat swabs, nasal washes, or expectorated sputum. It takes 48 to 72 hours for the cytopathic effects of virus to appear in tissue culture. Rapid diagnostic tests are also available. These include immunofluorescence (IF) assays, enzyme immunoassays (EIA), and polymerase chain reaction (PCR) and yield results as early as 15 minutes to 2 days.

In the United States, four medications are currently available for treatment: amantadine, oseltamivir, rimantadine, and zanamivir. Amantadine and rimantadine have activity against influenza type A, but not type B. Oseltamivir and zanamivir are neuraminidase inhibitors with activity against both influenza $A$ and $B$. If given within 48 hours of the onset of symptoms, these medications can reduce symptoms, fever, and viral shedding by about 1 day. However, none of these medications is effective in preventing serious complications of influenza such as those seen in the case of this patient.

Current treatments for influenza can only benefit a select group of patients who present early with uncomplicated disease. Therefore, prophylaxic immunization in appropriate patients remains the most effective means of controlling infection.

\section{References}

1. Anderson L, Besser R, Bridges C, Hajjeh R, Tablan, O. Guidelines for Preventing HealthCare-Associated Pneumonia. Available at www.CDC.gov. Accessed October 11, 2005.

2. Dolin, R. Influenza. In: Harrison's Principles of Internal Medicine, 15th ed, Braunwald, E, Fauci, AS, Kasper, DL, et al (Eds), McGraw Hill, New York, 2001, p. 1125.

3. Dolin, R. Clinical Manifestations and Diagnosis of Influenza in Adults. UpToDate version 13.2. Available at www.uptodate.com. Accessed October 11, 2005.

4. Harper, Scott A et al. Prevention and Control of Influenza: Recommendations of the Advisory Committee on Immunization Practices (ACIP) MMWR 29 July 2005; 54[RR08]:1-40

5. Treanor, JJ. Influenza virus. In: Principles and Practice of Infectious Diseases, 6th ed. Mandell, GL, Bennett, JE, Dolin, R, (Eds), Churchill Livingstone, Philadelphia, PA 2005, p.2060. 